\title{
Chirurgische Therapie der Gynäkomastie: Ein Algorithmus
}

\section{Surgical Treatment of Gynecomastia: An Algorithm}

Autoren

Institut

\author{
A. Wolter, T. Scholz, J. Diedrichson, J. Liebau
}

Klinik für Plastische und Ästhetische Chirurgie, Florence-Nightingale-Krankenhaus, Kaiserswerther Diakonie, Düsseldorf

\section{Schlüsselwörter \\ - Gynäkomastie \\ - subkutane Mastektomie \\ - Andromastektomie \\ - Algorithmus}

\section{Key words}

- gynecomastia

- subcutaneous mastectomy

- andromastectomy

- algorithm eingereicht 19.8.2012 akzeptiert $\quad 30.1 .2013$

\section{Bibliografie \\ DOI http://dx.doi.org/ 10.1055/s-0033-1334910 Handchir Mikrochir Plast Chir 2013; 45: 73-79 (c) Georg Thieme Verlag KG Stuttgart · New York ISSN 0722-1819}

Korrespondenzadresse Dr. med. Andreas Wolter Klinik für Plastische und Ästhetische Chirurgie Florence-NightingaleKrankenhaus Kaiserswerther Diakonie Kreuzbergstraße 79 40489 Düsseldorf a_wolter@gmx.de

\section{Zusammenfassung \\ $\nabla$}

Hintergrund: Die Gynäkomastie ist definiert als uni- oder bilaterale persistierende Brustdrüsenvergrößerung des Mannes, die von einer nur palpatorisch vergrößerten Brustdrüse bis hin zu einem weiblichen Thoraxerscheinungsbild reichen kann. In dieser Arbeit wird ein Algorithmus vorgestellt, der die Auswahl des geeigneten chirurgischen Verfahrens in Abhängigkeit von den morphologischen Aspekten des vorliegenden Befundes erleichtern soll.

Patienten und Methoden: Es handelt sich um eine retrospektive Analyse von 118 Patienten (217 männliche Mammae), die im Zeitraum von $01 / 2009$ bis $08 / 2012$ operiert wurden. Hierbei wurden die Ergebnisse von drei unterschiedlichen chirurgischen Therapieverfahren in Abhängigkeit von vier Schweregraden hinsichtlich der Outcome-Parameter Komplikationsrate, Patientenzufriedenheit, Brustwarzensensibilität und Notwendigkeit einer Korrekturoperation überprüft.

Ergebnisse: Bei einem geringen bis moderaten Ausgangsbefund ohne Hautüberschuss (Grad IIIa nach Simon) erfolgt die subkutane Mastektomie über eine semizirkuläre periareoläre Inzision sowie eine angleichende wasserstrahlassistierte Liposuktion. Bei vorhandenem Hautüberschuss und gut tastbarer Brustdrüse (Grad IIb) erfolgt zusätzlich eine periareoläre konzentrische Straffung. Bei den seltenen einer weiblichen Brust entsprechenden Befunden (Grad III) wird ein inframammärer Zugang mit kaudaler Stielung des Mamillen-Areolen-Komplexes verwendet. 167 Mammae (77\%) konnten über einen periareolären Zugang operiert werden, 40 Mammae $(18 \%)$ mit zusätzlicher periareolärer Straffung und zehn (5\%) über einen inframammären Zugang mit kaudaler Mamillenstielung. Die Komplikationsrate lag bei 4,1\%. Korrekturoperationen waren bei 17 Mammae (7,8\%) erforderlich. Eine Patientenbefragung ergab eine hohe Zufrieden-

\section{Abstract \\ $\nabla$}

Introductions: Gynecomastia is a persistent benign uni- or bilateral enlargement of the male breast ranging from small to excessive findings with marked skin redundancy. In this paper we introduce an algorithm to facilitate the selection of the appropriate surgical technique according to the presented morphological aspects.

Patients and Methods: The records of 118 patients ( 217 breasts) with gynecomastia from 01/2009 to $08 / 2012$ were retrospectively reviewed. The authors conducted three different surgical techniques depending on four severity grades. The outcome parameters complication rate, patient satisfaction with the aesthetic result, nipple sensitivity and the need to re-operate were observed and related to the employed technique.

Results: In 167 (77\%) breasts with moderate breast enlargement without skin redundancy (Grade I-Ila by Simon's classification) a subcutaneous semicircular periareolar mastectomy was performed in combination with water-jet assisted liposuction. In 40 (18\%) breasts with skin redundancy (Grade IIb) a circumferential mastopexy was performed additionally. An inferior pedicled mammaplasty was used in 10 (5\%) severe cases (Grade III). Complication rate was $4.1 \%$. Surgical corrections were necessary in 17 breasts (7.8\%). The patient survey revealed a high satisfaction level: $88 \%$ of the patients rated the aesthetic results as "very good" or "good", nipple sensitivity was rated as "very good" or "good" by $83 \%$. Conclusion: Surgical treatment of gynecomastia should ensure minimal scarring while respecting the aesthetic unit. The selection of the appropriate surgical method depends on the severity grade, the presence of skin redundancy and the volume of the male breast glandular tissue. The presented algorithm rarely leads to complications, is simple to perform and shows a high satisfaction rate and a preservation of the nipple sensitivity. 
heit: $88 \%$ der Patienten waren „sehr zufrieden“ bis „zufrieden“, die Sensibilität der Brustwarzen wurde von 83\% der Patienten als „sehr gut“ bis „gut“ bewertet.

Fazit: Die operative Behandlung der Gynäkomastie sollte möglichst narbensparend unter Respektierung der ästhetischen Einheit erfolgen. Hierbei ist die Wahl des operativen Verfahrens abhängig vom Schweregrad, dem Vorhandensein eines Hautüberschusses, sowie vom Volumen des zu resezierenden Brustdrüsengewebes. Der vorgestellte Algorithmus ist komplikationsarm, im Klinikalltag leicht anzuwenden und zeigt eine gute Bewahrung der Brustwarzensensibilität bei hoher Patientenzufriedenheit mit dem ästhetischen Ergebnis.

\section{Einleitung}

Die Gynäkomastie ist eine persistierende ein- oder beidseitige gutartige Vergrößerung der Brustdrüse des Mannes mit multifaktorieller Ätiologie. Fast ein Drittel aller Männer wird innerhalb der Lebenszeit mit den Symptomen einer Gynäkomastie konfrontiert. In der Mehrheit der Fälle ist die Ursache idiopathisch [1]. Physiologisch kann eine Gynäkomastie bei Neugeborenen (60-90\%), in der Pubertät (bis 65\%) und im Alter > 65 Jahre ( $>60 \%$ ) im Rahmen der Involutionsatrophie auftreten [2]. Während die sogenannte Pseudogynäkomastie sich durch eine reine Fettgewebsvermehrung bei adipösen Adoleszenten auszeichnet, beruht die echte, häufig mit Schmerzen verbundene Gynäkomastie, in der Regel auf Störungen im Hormonhaushalt. Hierbei ist ein Ungleichgewicht zwischen der östrogenen und androgenen Hormonkonzentration vorherrschend. Weitere Ursachen für ein vermehrtes Brustdrüsenwachstum beim Mann können durch paraneoplastische Prozesse (z.B. Hodentumor, Lungenkarzinom), metabolische Veränderungen (z.B. Nierenerkrankungen, Leberzirrhose, Hyperthyreose), medikamentös (z.B. Anabolika, Aldosteronantagonisten, Finasterid) oder durch Drogenmissbrauch (z.B. Heroin, Cannabis) bedingt sein.

Bei allen Patienten mit der Verdachtsdiagnose Gynäkomastie sollte immer eine ausführliche Anamnese und klinische Untersuchung inklusive der lokoregionären Lymphknotenstationen sowie eine Tumorausschlussdiagnostik in Form einer endokrinologischen bzw. urologischen Abklärung mit ggfs. bildgebender Diagnostik (Sono- und/oder Mammografie) erfolgen. Das sehr seltene, meist bei einseitigen Befunden auftretende Mammakarzinom sollte immer als Differenzialdiagnose eines vermehrten Brustdrüsenwachstums ausgeschlossen werden. Die Prävalenz für männlichen Brustkrebs liegt bei ca. $1 \%$ von allen Brustkrebsfällen und ist extrem selten bei einem Alter von weniger als 50 Jahren [3]. Die Sonografie ist als Mittel der Wahl zur Diagnostik eines verdächtigen Drüsenbefundes einsetzbar [4]. Im Falle eines suspekten Drüsenbefundes sollte eine histologische Abklärung in Erwägung gezogen werden.
Die Therapie der Wahl einer persistierenden und medikamentenresistenten Gynäkomastie ist in erster Linie die chirurgische Entfernung des überschüssigen Brustdrüsengewebes und ggfs. Hautmantels mit dem Ziel ein möglichst maskulines Thoraxerscheinungsbild bei narbensparendem Vorgehen zu erreichen. Hierzu sind verschiedene Methoden wie die Liposuktion, die subkutane Mastektomie über einen mamillären Zugang, die Kombination der genannten Verfahren sowie aufwendige Reduktionsplastiken möglich.

Webster klassifizierte die Gynäkomastie 1944 in drei Subtypen, abhängig von der Beschaffenheit des vermehrten Brustgewebes [5]. Diese Typen waren glandulär, glandulär mit Fettanteil und mit reinem Fettanteil ohne glanduläre Anteile. Simon unterteilte 1973 die Gynäkomastie nach der Größe und dem vorhandenen Hautüberschuss [6]. Je nach Ausmaß der Fehlanlage, die von einer klinisch nur palpatorisch feststellbaren Verdickung des Drüsenkörpers (Grad I) über eine bereits inspektorisch gut erkennbare Brustvergrößerung ohne Hautüberschuss (Grad IIa) bzw. mit Hautüberschuss (Grad IIb) bis zu einem einer weiblichen Brust entsprechenden Befund (Grad III) reicht, sollten unterschiedliche operative Maßnahmen eingesetzt werden.

Die Auswahl des geeigneten chirurgischen Verfahrens ist entscheidend für das ästhetische Endergebnis. Im klinischen Alltag kann daher ein Algorithmus hilfreich sein, der in Abhängigkeit von dem vorliegenden Gynäkomastiebefund die Entscheidung für das geeignete chirurgische Verfahren erleichtern soll. In der vorliegenden Arbeit wird ein befundabhängiger Algorithmus für die operative Behandlung der Gynäkomastie vorgestellt, basierend auf der Auswertung von 217 männlichen Mammae unterschiedlichen Schweregrades, die mit drei verschiedenen Techniken operiert wurden. Die qualitative Auswertung der Ergebnisse erfolgte in Bezug auf die Outcomeparameter Patientenzufriedenheit, Brustwarzensensibilität, Komplikationsrate und Notwendigkeit einer Korrekturoperation.

\section{Patienten und Methoden}

Es handelt sich um eine retrospektive Analyse von 118 Patienten (217 Mammae), bei denen eine chirurgische Therapie der Gynäkomastie durchgeführt worden ist. Präoperativ erfolgte bei allen Patienten zunächst eine ausführliche Anamneseerhebung, klinische Untersuchung sowie urologische bzw. endokrinologische Vorstellung zum Ausschluss einer anderen Genese der Brustdrüsenvergrößerung. Bei Vorliegen eines suspekten Befundes erfolgte zusätzlich die Vorstellung im angeschlossenen Brustzentrum zur Sono- bzw. Mammografie mit ggfs. Feinnadelbiopsie zur histologischen Abklärung. Als Basisdaten wurden festgehalten: Alter, OP-Dauer in Minuten, Verweildauer, Resektionsgewicht bzw. Fettaspirationsmenge der Liposuktion (s. 0 Tab. 1). Der von uns etablierte OP-Algorithmus ( $\bullet$ Abb. 1) diente uns als Leitfaden zur Auswahl des geeigneten chirurgischen Verfahrens

\begin{tabular}{|c|c|c|c|}
\hline & $\begin{array}{l}\text { Grad I/IIA } \\
(n=167,77 \%)\end{array}$ & $\begin{array}{l}\text { Grad IIB } \\
(n=40,18 \%)\end{array}$ & $\begin{array}{l}\text { Grad III } \\
(n=10,5 \%)\end{array}$ \\
\hline Alter & $26,5(12-69)$ & $38,3(14-70)$ & $41(18-78)$ \\
\hline OP-Dauer (min) & $65,6(25-123)$ & $91,3(45-136)$ & $118(78-160)$ \\
\hline VWD (d) & $3(1-4)$ & $3(2-6)$ & $5(3-10)$ \\
\hline Resektionsgewicht (g) & $28,9(3-104)$ & $36,7(5-116)$ & $305,5(187-430)$ \\
\hline WAL (ml) & $235(10-1000)$ & $354,4(50-1300)$ & - \\
\hline
\end{tabular}

Tab. 1 Demografische Basisdaten im Gesamtkollektiv (118 Pat. mammae: $n=217$ ) 


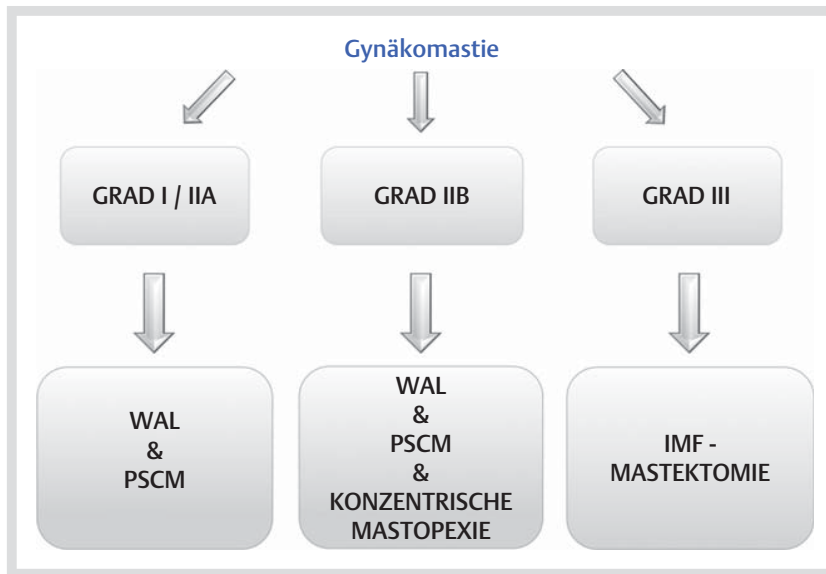

Abb. 1 OP-Algorithmus zum operativen stadienabhängigen Vorgehen bei Gynäkomastie. (WAL: Wasserstrahlassistierte Liposuktion, PSCM: periareoläre subkutane Mastektomie, IMF: Inframammärfalte).

Tab. 2 Gynäkomastie-Klassifikation nach Simon [6].

\begin{tabular}{|ll} 
Klassifikation der Gynäkomastie nach Simon [6] \\
Grad I & $\begin{array}{l}\text { Nur tastbare geringe Brustdrüsenvermehrung - kein } \\
\text { Hautüberschuss }\end{array}$ \\
\hline Grad lia & $\begin{array}{l}\text { Gut sichtbare, tastbare Brustdrüsenvermehrung ohne } \\
\text { Hautüberschuss }\end{array}$ \\
Grad lib & $\begin{array}{l}\text { Gut sichtbare, tastbare Brustdrüsenvermehrung mit } \\
\text { Hautüberschuss }\end{array}$ \\
Grad III & $\begin{array}{l}\text { Deutliche Brustdrüsenvermehrung mit Hautüberschuss } \\
\text { im Sinne eines weiblichen Brustdrüsenbefundes }\end{array}$
\end{tabular}
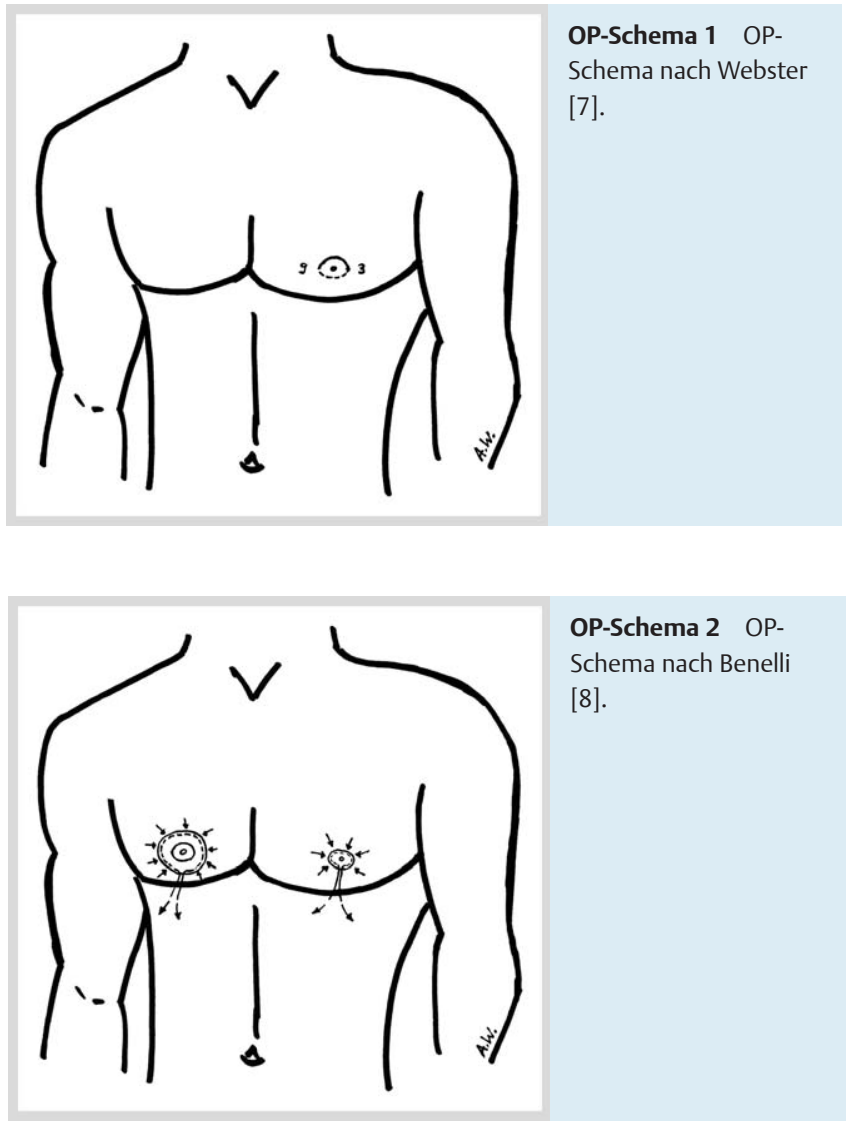

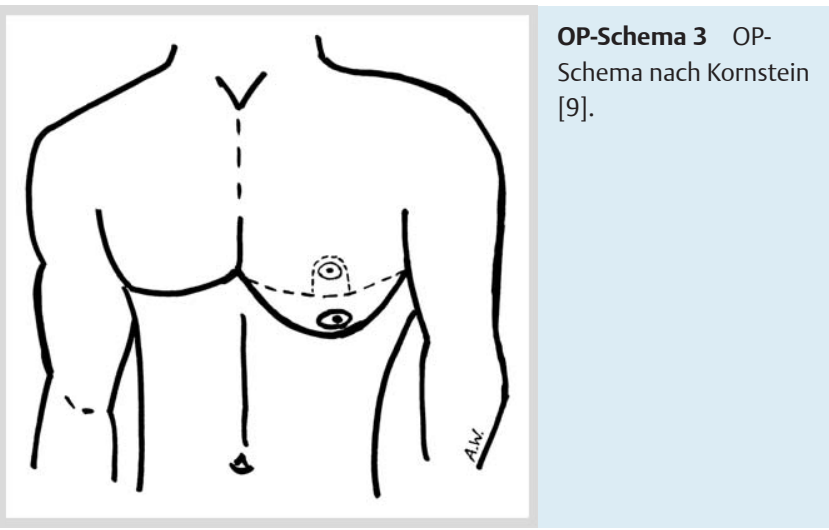

in Abhängigkeit vom vorliegenden Brustdrüsenbefund. Wir orientierten uns dabei an der bereits erwähnten Klassifikation nach Simon [6] (s. $\odot$ Tab. 2). Die Operationen erfolgten pro Brust durch einen Operateur mit Assistenz. Bei allen Patienten erfolgte präoperativ die Einzeichnung am aufrecht sitzenden Patienten. Alle Patienten erhielten eine Single-Shot Antibiose mit Cefuroxim 1,5 $\mathrm{g}$ intravenös. Bei einem geringgradigen Ausgangsbefund ohne Hautüberschuss (Grad I-IIa) erfolgte eine subkutane Mastektomie über eine periareoläre Inzision (PSCM) am Unterrand der Mamille nach Webster von 3 bis 9 Uhr [7] (s. ๑ OP-Schema 1). Der Drüsenkörper wurde hierbei schrittweise subkutan durch Lösung der Cooper-Ligamente und epifaszial vom Musculus pectoralis gelöst und über die ca. $2-3 \mathrm{~cm}$ lange Inzision exstirpiert. Ein Drüsenkörperrest von ca. 0,5-1 cm Dicke wurde immer unterhalb des Mamillenareolenkomplexes (MAK) erhalten, um eine ausreichende Durchblutung des MAK zu gewährleisten und ein ästhetisch unbefriedigendes Einsinken der Mamille oder Adhärenzen an der Pektoralisfaszie zu verhindern.

Zur angleichenden Liposuktion verwendeten wir ein wasserstrahlassistiertes Verfahren (WAL) der Firma human med ${ }^{\circledR}$, (1860 Crown Drive, Suite 1408, Dallas Texas 75234 USA) namens body-jet ${ }^{\circledR}$. Als Tumeszenzlösung wurde hierbei $1000 \mathrm{ml}$ Ringerlösung mit $1 \mathrm{ml}$ von 1: 1000 verdünntem Adrenalin verwendet. Die Zugangsinzisionen wurden in der Brustumschlagsfalte lateral angelegt. Die Kanülenstärke reichte hierbei von $3,5 \mathrm{~mm}$ bis $3,8 \mathrm{~mm}$.

Bei gut tastbarer Brustdrüse und vermehrt vorliegendem Hautüberschuss (Grad IIb) erfolgte zusätzlich zu dem vorgenannten Vorgehen eine periareoläre konzentrische Straffung im Sinne einer Benelli-Pexie [8] (s. ๑ OP-Schema 2). Hierbei erfolgte eine periareoläre Deepithelisierung des überschüssigen Hautmantels zirkulär am Außenrand der Mamille mit anschließender konzentrischer Straffung mittels eines 2/0 Mersilene-Fadens. Der Knoten wurde hierbei im subdermalen Fettgewebe versenkt, um das Risiko eines Fadengranuloms zu minimieren. Die Mamille wurde dann mit einem resorbierbaren monofilen Nahtmaterial zweireihig intrakutan vernäht.

Bei den seltenen drittgradigen Befunden, die einem weiblichen Brusttyp entsprachen, wurde ein inframammärer (IMF-) Zugang mit kaudaler Stielung des Mamillen-Areolen-Komplexes verwendet (IMF-Mastektomie). Wir lehnten uns dabei an die von Kornstein 1992 beschriebene Technik an [9] (s. O OP-Schema 3). Hierbei wurden zunächst die bereits bestehende Inframmärfalte sowie die kraniale Inzisionslinie so eingezeichnet, dass eine spindelförmige Resektionsfigur resultierte. Der kaudale Mamillen-Stiel wurde mit einer Breite von ca. $2-3 \mathrm{~cm}$ Größe eingezeichnet. Nach Überprüfung der Annäherung beider Resek- 
tionslinien und Sicherstellung der Spannungsfreiheit erfolgte zunächst die periareoläre zirkuläre Inzision um die Mamille. Dann erfolgte die Deepithelisierung der Mamillenstielfigur. Im Anschluss wurde die subkutane Mastektomie durch Durchtrennung der Cooper-Ligamente und Lösung von der Pektoralisfaszie unter Belassung eines ca. 1-2 cm starken Mamillenstiels durchgeführt. Anschließend erfolgte die Adaptierung der Hautränder unter Bedeckung des Mamillenstiels mit Positionierung der Naht in Höhe der Inframammärfalte. Nach Durchführung der Hautnaht erfolgte die Einzeichnung der neuen Mamillenposition am ca. $60^{\circ}$ aufrecht sitzenden Patienten. Im Anschluss erfolgte an dieser Stelle die Exzision der Haut und des subkutanen Fettpolsters, dann das Hervorluxieren des Mamillenstiels und schließlich die Fixierung der Mamille in ihrer neuen Position mittels eines zweireihig intrakutan vernähten monofilen Fadens.

Alle Patienten erhielten jeweils eine 12er Redondrainage, die erst ab einer Sekretionsmenge von $<30 \mathrm{ml} / 24$ Stunden wieder entfernt wurde. Die Wunden wurden mit Tegaderm ${ }^{\circledR}$-Folie verbunden, welche für eine Woche verblieb.

Das resezierte Brustdrüsengewebe wurde in allen Fällen der histologischen Untersuchung zugeführt. Zur Hämatom- und Seromprophylaxe sowie zur Förderung der Hautretraktion erhielten alle Patienten postoperativ ein Kompressionsmieder, welches sie für einen Zeitraum von 6 Wochen Tag und Nacht tragen mussten. Die Patienten wurden 14 Tage postoperativ zur Fadenentfernung, nach 3 Monaten sowie nach 12 Monaten nachuntersucht. Zur qualitativen Überprüfung unserer Ergebnisse erfolgte eine Evaluation der Komplikationsrate sowie der Anzahl von notwendigen Korrekturoperationen. Des Weiteren wurde sowohl telefonisch als auch im Rahmen der klinischen Nachuntersuchungen eine Patientenbefragung hinsichtlich der Zufriedenheit mit dem ästhetischen Ergebnis und dem subjektiven Grad der Brustwarzensensibilität durchgeführt. Für das ästhetische Ergebnis reichte der semiquantitative Score von 1 („sehr zufrieden“), 2 („zufrieden“), 3 („weniger zufrieden“) bis 4 („unzufrieden“). Die Brustwarzensensibilität konnte von 1 („sehr sensibel“), 2 („,sensibel“), 3 („mäßig sensibel“) bis 4 („nicht sensibel“) angegeben werden.

\section{Ergebnisse \\ $\nabla$}

Wir führten im Zeitraum von 01/2009 bis 08/2012 insgesamt 217 Operationen bei 118 Gynäkomastie-Patienten durch (s. $\odot$ Tab. 1). Bei 19 Patienten (9\%) lag ein einseitiger Befund vor. Das mittlere Alter lag bei 28,9 Jahren (12-78 J.). 167 Mammae (77\%) konnten narbensparend über einen semizirkulären periareolären Zugang (Grad I-IIa) operiert werden, 40 Mammae (18\%) mit zusätzlicher periareolärer Straffung (Grad IIb) und 10 Mammae (5\%) über einen inframammären Zugang mit kaudaler Mamillenstielung (Grad III) (s. auch Patientenbeispielfälle - Abb. 2-5).

Insgesamt traten 9 Komplikationen (4,1\%) auf. Davon waren 7 revisionsbedürftige Nachblutungen (6 davon in der Grad I/IIaGruppe und eine in der Grad III-Gruppe) sowie eine oberflächliche Epitheliolyse in der Grad IIb-Gruppe und eine oberflächliche Wundinfektion in der Grad III-Gruppe (s. ๑ Tab. 3).

Im Rahmen der Verlaufskontrollen konnten insgesamt 101 von 118 (85,6\%) befragt bzw. nachuntersucht werden. Dabei waren 33 Patienten (33\%) mit dem ästhetischen Ergebnis „sehr zufrieden“ (Score = 1), 56 Patienten (55\%) „Zufrieden“ (Score=2), 11 Patienten $(11 \%)$,weniger zufrieden“ (Score $=3)$ und nur ein Pa- tient (1\%) war „nicht zufrieden“ (Score =4). Bezüglich der Sensibilitität der Brustwarzen ergaben sich folgende Ergebnisse: 26 Patienten (26\%) gaben eine „sehr gute“ Sensibilität (Score=1) an, 58 Patienten (57\%) eine „gute“ (Score=2), 16 Patienten (16\%) eine „mäßige“ (Score =3) und 1 Patient (1\%) „keine“ Sensibilität. Die Verteilung der genannten Outcome-Parameter bezogen auf die jeweilige Operationstechnik ist in 0 Tab. 3 abgebildet.

In allen Gruppen zeigten sich sehr gute Ergebnisse. Die Technik der periareolären Mastektomie in Kombination mit WAL bei Grad I/Ila-Befunden stuften 69 von 77 Patienten (90\%) als „sehr gut“ bis „gut“ ein, die zusätzliche periareoläre Straffung 16 von 19 Patienten (84\%) als „sehr gut“ bis „gut“ und die IMF-Mastektomie 4 von 5 Patienten mit „gutem“ Ergebnis (80\%). Vergleichbar sehr gute Ergebnisse zeigten sich auch in Bezug auf die Sensibilität der Brustwarzen (s. $\bullet$ Tab. 3).

In Bezug auf eine Reoperation zur ästhetischen Optimierung des Endergebnisses waren im Verlauf bei 17 von insgesamt 217 operierten Mammae (7,8\%) Korrekturen erforderlich. In der Grad I/ Ila-Gruppe war bei 12 von 167 Mammae (7,2\%) eine Narbenkorrektur bzw. in 2 Fällen eine Korrektur einer eingezogenen Brust-

\section{Abbildungen mit operativen Fallbeispielen unterschiedlicher Schweregrade der Gynäkomastie:}
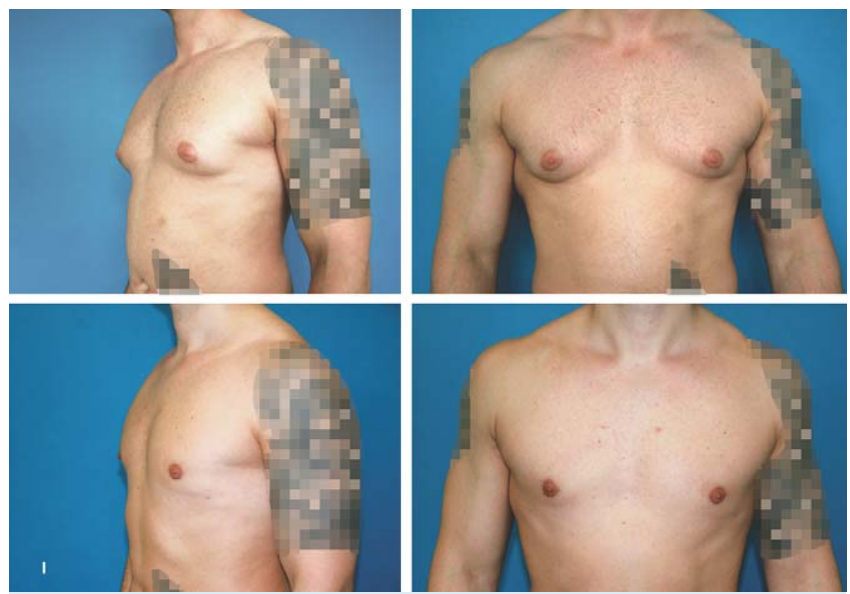

Abb. 2 Grad I: 26-jähriger Patient präoperativ (obere Reihe) und postoperativ (untere Reihe) nach WAL und PSCM (Resektionsgewicht rechts $81 \mathrm{~g}$, links $46 \mathrm{~g}$, Fettaspirationsmenge $50 \mathrm{ml} /$ Seite).
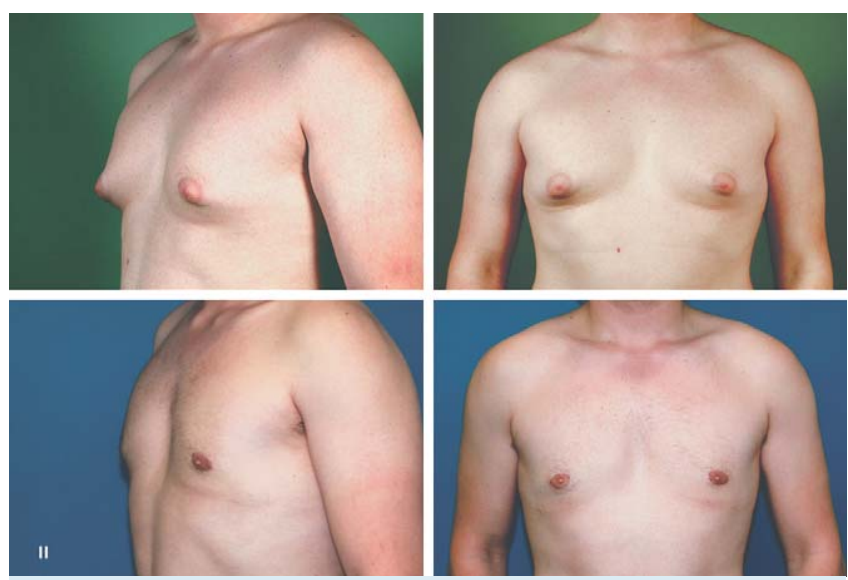

Abb. 3 Grad IIA: 34-jähriger Patient präoperativ (obere Reihe) und postoperativ (untere Reihe) nach WAL und PSCM (Resektionsgewicht rechts: $25 \mathrm{~g}$, links $31 \mathrm{~g}$, WAL-Fettaspirationsmenge rechts $300 \mathrm{ml}$, links $350 \mathrm{ml}$ ). 

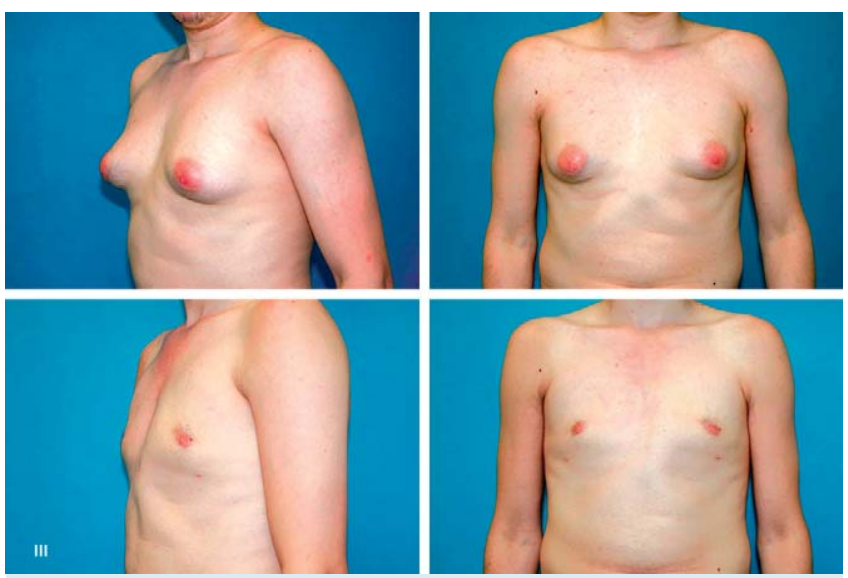

Abb. 4 Grad IIB: 19-jähriger Patient präoperativ (obere Reihe) und postoperativ (untere Reihe) nach WAL, PSCM u. zirkulärer konzentrischer Straffung (Resektionsgewicht rechts: $94 \mathrm{~g}$, links $116 \mathrm{~g}$, WAL-Fettaspirationsmenge: $150 \mathrm{ml} /$ Seite).
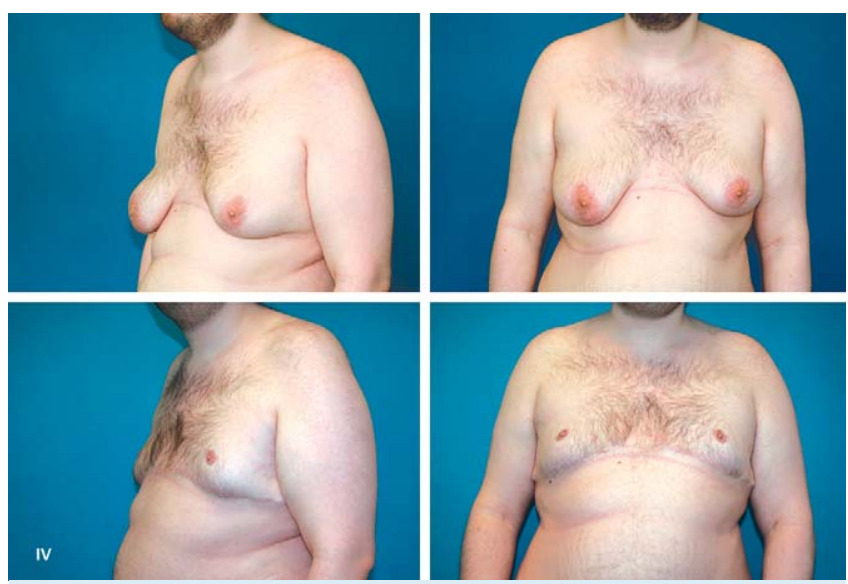

Abb. 5 Grad III: 26-jähriger Patient präoperativ (obere Reihe) und 1 Jahr postoperativ (untere Reihe) nach subkutaner Mastektomie mit kaudal gestielten Mamillen (Resektionsgewicht rechts: 379 g, links: 346 g); zwischenzeitliche Gewichtszunahme des Patienten um 15 kg KG durch Einnahme von Quetiapin (Seroquel). (WAL: Wasserstrahlassistierte Liposuktion, PSCM: periareoläre subkutane Mastektomie). warze erforderlich. In der Grad IIb-Gruppe wurden 3 von 40 Mammae (7,5\%) einer Narbenkorrektur bzw. Nachstraffung des Hautmantels unterzogen und in der Grad III-Gruppe war bei 2 von 10 Mammae (20\%) eine Narbenkorrektur erforderlich. (s. Tab. 3). In der histologischen Aufarbeitung zeigte sich in keinem Fall der Nachweis von Malignität.

\section{Diskussion}

\section{$\nabla$}

Zur Therapie der Gynäkomastie sind in der Literatur zahlreiche Veröffentlichungen zu finden. Eine medikamentöse Therapie kann als konservative Therapieoption in Erwägung gezogen werden. Hier kommen Antiöstrogene wie z.B. Tamoxifen oder andere hormonmodulierende Substanzen zur Anwendung. Die Erfolgsaussichten des konservativen Weges sind in der Regel jedoch gering. Die chirurgische Entfernung im Sinne einer subkutanen Mastektomie ist bei korrekter medizinischer Indikationsstellung die Methode der Wahl. Präoperativ ist die interdisziplinäre Differenzialdiagnostik (Urologie, Endokrinologie, Radiologie, Pathologie) zum Ausschluss einer anderen Genese des vermehrten Brustdrüsenwachstums obligat.

Das Spektrum der modernen chirurgischen Therapieoptionen der Gynäkomastie variiert heute von minimal-invasiven Techniken wie der Liposuktion, einer direkten chirurgischen Exstirpation, einer Kombination aus beiden Verfahren bis hin zu ausgedehnten Reduktionsverfahren bei hochgradigen Befunden. Das Ziel ist hierbei nicht nur die Schaffung eines ästhetischen maskulinen Thoraxerscheinungsbildes und Beschwerdefreiheit, sondern auch die Vermeidung von ausgedehnten, zum Teil entstellenden Narben sowie eine geringe Morbiditätsrate.

Erstmals wurde die operative Korrektur der Gynäkomastie im 7. Jahrhundert nach Christus von dem italienischen Chirurgen Paulus von Aigina beschrieben, der eine Drüsenentfernung über einen halbmondförmigen Schnitt durchführte [10]. Seither wurde eine Vielzahl von möglichen Operationstechniken in der Literatur beschrieben. Heutzutage verwenden die meisten Operateure den 1946 von Webster beschriebenen semizirkulär-periareolär inferioren Zugangsweg zur subkutanen Entfernung des Drüsenkörpers [7]. Ergänzend wird oft eine Liposuktion durch-

Tab. 3 Aufstellung der Outcomeparameter in Bezug auf die 3 angewandten OP-Techniken bzw. Gynäkomastieschweregrade.

\begin{tabular}{|c|c|c|c|c|}
\hline & $\begin{array}{l}\text { Gesamt } \\
n=101\end{array}$ & $\begin{array}{l}\text { PSCM \& WAL } \\
\text { (Grad I/IIA) } \\
n=77\end{array}$ & $\begin{array}{l}\text { PSCM, WAL \& konz. } \\
\text { Pexie (Grad IIB) } \\
n=19\end{array}$ & $\begin{array}{l}\text { IMF - Mastektomie } \\
\text { (Grad III) } \\
n=5\end{array}$ \\
\hline \multicolumn{5}{|l|}{ Patientenzufriedenheit } \\
\hline $1=$ sehr zufrieden & $33(33 \%)$ & 28 & 5 & 0 \\
\hline 2 = zufrieden & $56(55 \%)$ & 41 & 11 & 4 \\
\hline $3=$ weniger zufrieden & $11(11 \%)$ & 7 & 3 & 1 \\
\hline 4 = unzufrieden & $1(1 \%)$ & 1 & 0 & 0 \\
\hline \multicolumn{5}{|l|}{ Brustwarzensensibilität } \\
\hline 1 = sehr sensibel & $26(26 \%)$ & 20 & 6 & 0 \\
\hline $2=$ sensibel & $58(57 \%)$ & 46 & 9 & 3 \\
\hline 3= mäßig sensibel & $16(16 \%)$ & 10 & 4 & 2 \\
\hline $4=$ nicht sensibel & $1(1 \%)$ & 0 & 1 & 0 \\
\hline Komplikationen von 217 Mammae & & 6 & 1 & 2 \\
\hline Korrekturoperationen von 217 Mammae & & 12 & 3 & 2 \\
\hline
\end{tabular}

WAL: Wasserstrahlassistierte Liposuktion, PSCM: periareoläre subkutane Mastektomie, IMF Inframammärfalte 
geführt. Die Fettabsaugung kann hierbei ultraschall-, wasserstrahlassistiert oder lasergestützt sein [11-13].

Wichtig zu erwähnen ist hierbei, dass eine alleinige Liposuktion nur bei einer reinen Fettgewebsvermehrung (Lipomastie) im Rahmen einer Pseudogynäkomastie durchgeführt werden sollte, da es bei Vorliegen eines Drüsenkörpers im Rahmen der „echten“ Gynäkomastie zu Versprengungen des Brustdrüsengewebes und somit zu unerwünschten Nebenwirkungen und einer hohen Rezidivneigung kommen kann [14].

Grundsätzlich ist bei plastisch-chirurgischen Operationen immer ein möglichst narbensparendes Vorgehen zu favorisieren. Daher ist auch ein Trend zu beobachten, dass früher benutzte Verfahren wie z.B. die T-Technik heute so gut wie nicht mehr verwendet werden. Der Trend geht zu minimal-invasivem Vorgehen. Hier ist auch die von Morselli und Hammond beschriebene narbensparende sogenannte „pull-through“-Technik zu erwähnen, die vor allem bei Befunden angewandt werden kann, bei denen sich die Brustdrüsenvermehrung auf den subareolären Bereich beschränkt $[15,16]$. Hierbei wird der Drüsenkörper nach erfolgter Liposuktion über eine ca. $0,5-1 \mathrm{~cm}$ große Inzision am Mamillenaussenrand schrittweise exstirpiert. Neuerlich beschrieben ist auch die Verwendung eines arthroskopischen Knorpelshavers, mit dessen Hilfe der Drüsenbefund schrittweise durch Abradierung reduziert wird [17,18].

Diagnostische Algorithmen sowie konservative Therapieempfehlungen der Gynäkomastie findet man in der Literatur häufig [19], operative Schemata mit algorithmischer befundabhängiger Darstellung findet man nur wenige [14,18, 20,21]. In der vorliegenden Arbeit wird ein befundabhängiger OP-Algorithmus vorgestellt (s. A Abb. 1), der es ermöglicht, möglichst narbensparend in Abhängigkeit vom Schweregrad, dem Vorhandensein eines Hautüberschusses sowie vom Volumen des zu resezierenden Brustdrüsengewebes alle Ausprägungen der Gynäkomastie durch drei operative Verfahren adäquat zu behandeln. Der von Cordova et al. beschriebene Algorithmus untersuchte nicht jede Brust separat, wandte vier OP-Techniken an und überprüfte die Ergebnisse zwar anhand von Komplikationsrate und Reoperationsrate, aber nicht anhand der Patientenzufriedenheit und Brustwarzensensibilität. Li et al. untersuchten über einen Zeitraum von 10 Jahren nur eine kleine Fallzahl von 41 Patienten mit 75 Operationen [14]. Hierbei zeigte sich eine Komplikationsrate von $12,1 \%$. Die Korrekturrate sowie die Brustwarzensensibilität wurden nicht untersucht, im Algorithmus wurde nicht zwischen Grad IIa und IIb unterschieden. Fruhstorfer et al. verwandten in ihrem Algorithmus bei Grad III-Befunden eine Straffung nach Lejour mit einer sehr auffälligen vertikalen Narbenbildung [21]. Postoperativ verbliebener Hautüberschuss und inakzeptable ästhetische Ergebnisse stellten bei Petty et al. durch alleinigen Einsatz von Liposuktion und eines arthroskopischen Knorpelshavers ebenfalls ein häufiges Problem dar, insbesondere bei sehr festem Drüsengewebe und höhergradigen Befunden (Grad III) [18]. Des Weiteren ist das Rezidivrisiko als erhöht einzustufen bei alleiniger Verwendung der vorgenannten Technik nach Petty durch eine mögliche Versprengung von Drüsenkörperanteilen.

Der Hauptteil unseres Patientengutes (77\%, 167 Mammae) wurde narbensparend über einen periareolären inferioren Zugang in Kombination mit wasserstrahlassistierter Liposuktion (WAL) operiert. Der Zugangsweg nach Webster lässt eine Exstirpation des Drüsenkörpers unter Schonung des Brustwarzenkomplexes bei sehr unauffälliger Narbenbildung zu. Das WAL-Verfahren zeichnet sich durch eine Reduzierung der postoperativen Komplikationen wie Hämatome, postoperative Schmerzen, ödema- töse Schwellungen, Entzündungsreaktionen sowie Sensibilitätsstörungen aus [12,22]. Bei dieser sehr gewebsschonenden und komplikationsarmen Technik ist es möglich simultan die Tumeszenzlösung bestehend aus Ringerlösung und Adrenalin zu infiltrieren und gleichzeitig die Fettzellen mit einem Wasserstrahl aus dem umgebenden Gewebe zu lösen und abzusaugen. Des Weiteren führt diese Technik analog zu den gängigen Liposuktionsverfahren zu einer erhöhten Hautretraktion postinterventionell nach ca. 2-6 Monaten [12,22]. Gerade bei jungen Patienten mit guter Hautqualität und damit erhöhter Retraktionsfähigkeit der Haut ist dadurch in vielen Fällen mit grenzwertigen Befunden (Grad IIa/IIb) eine zusätzliche Hautmantelstraffung im Verlauf häufig nicht erforderlich.

Die meisten Befunde (Grad I-Ila) sind über eine Kombination aus subkutaner Mastektomie und Liposuktion gut therapierbar. Bei einem vorliegenden Hautüberschuss (Grad IIb) ist in vielen Fällen eine Straffungsoperation des überschüssigen Hautmantels jedoch unumgänglich. Benelli und Huang beschrieben hier bereits mögliche Verfahren [8,23]. Die „round-block technique“ von Benelli zeigte im Bereich der Mammachirurgie ausgezeichnete Ergebnisse und lässt auch im Bereich der Gynäkomastie sehr gute Ergebnisse bei narbensparendem Vorgehen zu.

In der vorliegenden Arbeit ließen sich bei 40 Mammae mit vermehrtem Hautüberschuss (18\%) über die konzentrische Straffungstechnik nach Benelli in Bezug auf alle vier Outcomeparameter sehr gute Ergebnisse erzielen.

Hochgradige Befunde (Grad III) mit häufig vorhandener Lokalisierung des Mamillenareolenkomplexes in Höhe oder unterhalb der Inframammärfalte, aber auch ältere Patienten mit verminderter Hautretraktionsfähigkeit erfordern in vielen Fällen eine extraareoläre Vorgehensweise mit entsprechenden Straffungsund Reduktionsverfahren $[8,9,23,24]$. Bei extremen Formen und zusätzlichen Komorbiditäten ist auch eine Mastektomie in Kombination mit einer freien Mamillentransplantation beschrieben [25]. In England wird die von Letterman 1972 beschriebene Technik häufig angewandt [24]. Hierbei wird ein Lförmiger Zugang zur Drüsenexstirpation und medial gestielter Mamillentransposition verwandt.

In unserer Klinik favorisieren wir die von Kornstein [9] beschriebene kaudale Stielung des Mamillen-Areolenkomplexes (MAK). Trotz Vorliegen eines sehr ausgeprägten Befundes kann hierbei durch die kaudale Gewebestielung des MAK die Brustwarzensensibilität erhalten werden. Die Narbe kommt in der Inframammärfalte zu liegen (IMF-Mastektomie) und folgt somit relativ unauffällig dem natürlichen Erscheinungsbild der männlichen Thoraxkontur. Bei anderen Techniken wie dem invertierten T, I- oder L-Technik ist die Narbe in nicht-natürlichem Verlauf häufig sehr offensichtlich auf der Brust gelegen.

Die Komplikationsrate lag insgesamt bei nur 4,1\%, die Korrekturrate bei nur $8 \%$ und liegt dabei im unteren Durchschnitt der in der Literatur zu findenden Ergebnisse, bei denen Komplikationsraten von 15,5-46\% zu finden sind [14,26].

Der vorgestellte Algorithmus wurde anhand der Auswertung von Ergebnissen entwickelt, die sich durch die Anwendung von drei unterschiedlichen Therapieverfahren bei vier unterschiedlichen Schweregraden (s. $\bullet$ Abb. 1) ergaben. Die vorgestellte Arbeit zeichnet sich nicht nur durch eine in der Literatur sehr selten zu findende besonders hohe Fallzahl (217 männliche Mammae innerhalb von nur 44 Monaten) aus, sondern auch durch eine qualitative Überprüfung der Ergebnisse in Bezug auf vier Outcome-Parameter: Patientenzufriedenheit, Brustwarzensensibilität, Komplikationsrate und Notwendigkeit von Korrekturo- 
perationen. Dabei beschränken wir uns auf drei OperationsTechniken, die in allen Bereichen der genannten Parameter, unabhängig von jeweiligen Ausgangsbefund und Schweregrad, überwiegend sehr gute bis gute Ergebnisse erreichen konnten. Darüber hinaus lässt sich der Algorithmus aufgrund seiner klaren Darstellung leicht in den klinischen Alltag übertragen. In unserer Abteilung findet der hier vorgestellte OP-Algorithmus in leichter Abwandlung darüber hinaus Anwendung bei der Behandlung von transsexuellen Patienten mit dem Wunsch der geschlechtsangleichenden Operation von der Frau zum Mann. Hierbei wird im Rahmen der subkutanen Mastektomie ein analoges narbensparendes Vorgehen angestrebt.

\section{Schlussfolgerung}

$\nabla$

Vor einer jeden Operation bei Symptomen einer Gynäkomastie ist der interdisziplinäre Ausschluss einer anderen Genese obligat. Bei der operativen Korrektur der Gynäkomastie ist ein möglichst narbensparendes Vorgehen anzustreben. Das operative Vorgehen hängt hierbei vom Schweregrad, vom Ausmaß des Hautüberschusses sowie vom Volumen des zu resezierenden Brustdrüsengewebes ab. Der hier vorgestellte im klinischen Alltag leicht anzuwendende OP-Algorithmus ermöglicht die Auswahl aus drei operativen Verfahren und zeigt dabei sehr gute Ergebnisse mit hoher Patientenzufriedenheit, geringer Komplikations- und Korrekturoperationsrate bei Erhaltung der Brustwarzensensibilität.

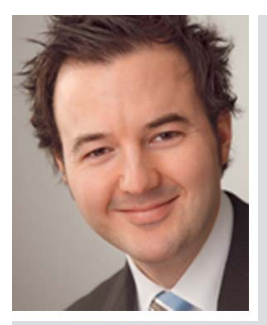

Andreas Wolter

Geboren am 29.03.1979 in Stolberg (Rhld.). 1999-2006 Studium der Humanmedizin an der Rheinisch-Westfälischen Technischen Hochschule (RWTH) Aachen. 2006-2008 Absolvierung des „common trunk“ in der Abteilung für Viszeral-, Gefäß- und Transplantationschirurgie des Klinikum Köln-Merheim, Lehrkrankenhaus der Universität Witten-Herdecke (Prof. Dr. med. M.M. Heiss). 2008-2010 Assistenzarzt in der Abteilung für Thoraxchirurgie der Lungenklinik Köln-Merheim, Lehrkrankenhaus der Universität Witten-Herdecke (Prof. Dr. med. E. Stoelben). Promotion 2010 in der Klinik für Plastische, Hand- und Verbrennungschirurgie des Universitätsklinikum der RWTH Aachen (Univ.-Prof. Dr. Dr. med. Prof. h.c. mult. N. Pallua). Seit 2010 in Facharztweiterbildung in der Klinik für Plastische und Ästhetische Chirurgie des Florence-Nightingale-Krankenhauses, Kaiserswerther Diakonie (Prof. Dr. med. J. Liebau).

\section{Literatur}

1 Berger A, Simma W, Fische P. Kerngeschlechtsbestimmungen bei der idiopathischen Gynäkomastie. Wien Klin Wochenschr 1972; 84: 699-701

2 Braunstein GD. Gynecomastia. N Engl J Med 1993; 328: 490-495

3 Sasco AJ, Lowenfels AB, Pasker-De Jonc P. Epidemiology of male breast cancer. A meta-analysis of published case - control studies and discussion of selected etiologic factors. Int J Cancer 1993; 53: 538-549

4 Adibelli $Z$ H, Oztekin 0 , Postaci $H$ et al. The Diagnostic Accuracy of Mammography and Ultrasound in the Evaluation of Male Breast Disease: A New Algorithm. Breast Care (Basel) 2009; 4: 255-259

5 Webster GV. Gynecomastia in the Navy. Mil Surg 1944; 95: 375-379

6 Simon BE, Hoffmann S, Kahn S. Classification and surgical correction of gynecomastia. Plast Reconstr Surg 1973; 51: 48-56

7 Webster JP. Mastectomy for gynecomastia through a semicircular intra-areolar incision. Ann Surg 1946; 124: 557-575

8 Benelli L. A new periareolar mammoplasty: the „round block“ technique. Aesthetic Plast Surg 1990; 14: 93-100

9 Kornstein $A N$, Cinelli $P B$. Inferior pedicle reduction technique for larger forms of gynecomastia. Aesthetic Plast Surg 1992; 16: 331-335

10 Aegineta $P$. On male breast resembling the female. In: Adams F, Hrsg. The seven books of Paulus Aegineta, 46. Vol 2, Book 4. Sydenham Society; London: 1846; 334-335

11 Rohrich RJ, Ha RY, Kenkel JM et al. Classification and management of gynecomastia: defining the role of ultrasound-assisted liposuction. Plast Reconstr Surg 2003; 111: 909-923

12 Sasaki GH. Water-assisted liposuction for body contouring and lipoharvesting: safety and efficacy in 41 consecutive patients. Aesthet Surg J 2011; 31: 76-88

13 Trelles MA, Mordon SR, Bonanad E et al. Laser-assisted lipolysis in the treatment of gynecomastia: a prospective study in 28 patients. Lasers Med Sci 2012 [Epub ahead of print]

14 Li CC, Fu JP, Chang SC et al. Surgical Treatment of Gynecomastia: Complications and Outcomes. Ann Plast Surg 2011 [Epub ahead of print]

15 Morselli PG. „Pull-through“: a new technique for breast reduction in gynecomastia. Plast Reconstr Surg 1996; 97: 450-454

16 Hammond DC, Arnold JF, Simon AM et al. Combined use of ultrasonic liposuction with the pull-through technique for the treatment of gynecomastia. Plast Reconstr Surg 2003; 112: 891-895; discussion 896-897

17 Lee JH, Kim IK, Kim TG et al. Surgical Correction of Gynecomastia with Minimal Scarring. Aesthetic Plast Surg. 2012 [Epub ahead of print]

18 Petty PM, Solomon M, Buchel EW et al. Gynecomastia: evolving paradigm of management and comparison of techniques. Plast Reconstr Surg 2010; 125: 1301-1308

19 Rahmani S, Turton P, Shaaban A et al. Overview of gynecomastia in the modern era and the Leeds Gynaecomastia Investigation algorithm. Breast J 2011; 17: 246-255

20 Cordova A, Moschella F. Algorithm for clinical evaluation and surgical treatment of gynecomastia. J Plast Reconstr Aesthet Surg 2008; 61: 41-49

21 Fruhstorfer BH, Malata CM. A systematic approach to the surgical treatment of gynecomastia. Br J Plast Surg 2003; 56: 237-246

22 Araco A, Gravante G, Araco F et al. Comparison of power water-assisted and traditional liposuction: a prospective randomized trial of postoperative pain. Aesthetic Plast Surg 2007; 31: 259-265

23 Huang TT, Hidalgo JE, Lewis SR. A circumareolar approach in surgical management of gynecomastia. Plast Reconstr Surg 1982; 69: 35-40

24 Letterman $G$, Schurter $M$. Surgical correction of massive gynecomastia. Plast Reconstr Surg 1972; 49: 259-262

25 Wray RC, Hoopes JE, Davis GM. Correction of extreme gynecomastia. Br J Plast Surg 1974; 27: 39

26 Steele SR, Martin MJ, Place RJ. Gynecomastia: complications of the subcutaneous mastectomy. Ann Surg 2002; 68: 210-213 